\title{
Fulminant amoebic colitis: the role of surgery and the operative approach
}

\author{
Mark Wagener ${ }^{1 *}$ and Maheshwar Naidoo ${ }^{2}$ \\ ${ }^{1}$ Department of Paediatric Surgery, Inkosi Albert Luthuli Central Hospital, KwaZulu Natal, South Africa \\ ${ }^{2}$ Head of Deparment, General Surgery, Ngwelezane Hospital, KwaZulu Natal, South Africa
}

\begin{abstract}
Fulminant amoebic colitis is rare and has a high mortality. The role of surgery in the management of this condition is examined using examples from two cases presenting one year apart at Ngwelezane Hospital in KwaZulu Natal, South Africa. Both patients presented in an extremely unwell state with distended, peritonitic abdomens. Evidence from these cases confirms findings from others in the literature that the best management for fulminant amoebic colitis associated with extensive involvement, multiple perforations and gross contamination is total or subtotal colectomy and exteriorization of proximal bowel with or without mucous fistula.
\end{abstract}

\section{Introduction}

Amoebiasis is the third leading cause of death from parasitic infection worldwide [1]. The first two being malaria and schistosomiasis. Most people exposed to Entamoeba histolytica are asymptomatic. Amoebic cysts are ingested, these breakdown to release trophozoites in the small intestine which migrate to the colon, where they may be indolent, cause amoebic colitis or invade into the vasculature and enter the liver via the portal venous system where they may form an abscess. Cysts reform in the colon, are excreted in faeces and spread to others by the faeco-oral route. Endemic areas such as ours are characterized by tropical or subtropical climate as well as widespread poverty with resultant poor sanitation enabling faeco-oral spread.

Amoebic colitis is typically described as presenting with cramping abdominal pain, watery or bloody diarrhoea, anorexia and fever. Microscopic examination of stool samples and finding amoebic trophozoites confirms the diagnosis but this has a low sensitivity (25$60 \%)$. Positive serology represents current or previous infection and has a good negative predictive value. Antigen detection assays in stool samples is sensitive and specific but is not available in all laboratories. Treatment is metronidazole. Certainly for uncomplicated amoebic colitis, surgery is contra-indicated: bowel resembles "wet blotting paper" is friable and perforates easily if handled.

A variant exists, however, known as fulminant amoebic colitis. Presented here are two cases occurring 1 year apart at Ngwelezane Hospital in KwaZulu Natal, South Africa. Patients developed inflammation and necrosis along the full length of the colon with multiple sites of perforation and faecal peritonitis.

Both patients were children from the local population with no history of travel. Both gave a relatively short history of symptoms and arrived severely unwell with signs of generalized peritonitis.

\section{Case 1}

A 15 year old boy presented with a 3 day history of abdominal pain, associated with vomiting and generalized weakness. There was no history of diarrhoea or rectal bleeding. The patient was previously well. Examination revealed temperature of $38.7^{\circ} \mathrm{C}$, Pulse $141 \mathrm{bpm}$, Blood pressure $108 / 68 \mathrm{~mm} \mathrm{Hg}$. The patient was toxic looking. Abdominal examination revealed tense distended abdomen with generalised peritonitis.

Investigations showed a leucocytosis of $19.9 \times 10^{9} / \mathrm{L}$ and haemoglobin of $9.0 \mathrm{~g} / \mathrm{dL}$. Urea, creatinine and serum electrolytes were normal. Albumin was $13 \mathrm{~g} / \mathrm{L}$. Abdominal x-ray showed dilated loops of small bowel. There was no free gas on erect chest $\mathrm{x}$-ray. The patient was resuscitated and then taken for an emergency laparotomy. At operation the patient had free stool in peritoneum; perforations to caecum, sigmoid colon and rectum; and a pelvic abscess. Perforations were repaired, rectal biopsy was taken, peritoneal lavage performed and a loop ileostomy done.

In the immediate post-operative period, while still on the operating table awaiting transfer to ICU patient went into cardiopulmonary arrest. An unsuccessful resuscitation attempt followed and the patient died. The histopathological analysis of the specimen revealed ulcerated colonic tissue with granulation tissue and acute inflammatory exudate. Transmurally amoebic trophozoites were found.

\section{Case 2}

A 5 year old girl presented with 2 week history of diarrhoea. She had deteriorated over a 24 hour period with: worsening abdominal pain, abdominal distention and anorexia. There was no associated vomiting. Diarrhoea was watery, with no blood. She had no past medical or surgical history. She was HIV exposed, but HIV PCR done in infancy

Correspondence to: Dr. Mark Wagener, Registrar, Department of Paediatric Surgery, Inkosi Albert Luthuli Central Hospital, KwaZulu Natal, South Africa; E-mail: Mark.wagener2@gmail.com

Key words: Fulminant amoebic colitis, entamoeba histolytica, amoebiasis

Received: August 07, 2016; Accepted: August 29, 2016; Published: August 31, 2016 
was negative. Rapid HIV test on admission was negative. The child was in severe discomfort and acutely unwell. She had a temperature of $36.7^{\circ} \mathrm{C}$ and Pulse $167 \mathrm{bpm}$.

The abdomen was distended with generalized peritonism. Laboratory investigations revealed $\mathrm{Na} 129 \mathrm{mmol} / \mathrm{L}$, with other electrolytes, urea and creatinine being normal. A leukopaenia of 5.25 $\times 10^{9} / \mathrm{L}$ and haemoglobin of $10.9 \mathrm{~g} / \mathrm{dL}$. Albumin was $12 \mathrm{~g} / \mathrm{L}$. Blood gas analysis showed $\mathrm{pH} 7.38, \mathrm{pCO}_{2} 3.1 \mathrm{kPa}$, standard $\mathrm{HCO}_{3} 17.2 \mathrm{mmol} / \mathrm{L}$ and Base excess of -9.8 .

After a period of resuscitation we proceeded with laparotomy. At laparotomy the patient had generalized peritoneal contamination with free faeces and pus. There was inflammation and multiple perforations of colon from caecum to proximal sigmoid. Distal sigmoid appeared healthy. A sub-total colectomy was performed. Distal sigmoid stump was over sewn and an end ileostomy formed. A thorough was-out was done. Post operatively the patient went to paediatric ICU. Antibiotics given were ceftriaxone and metronidazole. She was extubated day 1 post operatively and discharged day 5 from ICU. The patient developed superficial wound sepsis which was successfully managed with daily dressings.

She was discharged from hospital after a total hospital stay of 31 days with an aim to re-admit and do a laparotomy to restore continuity within 6 months. Histopathological analysis showed diseased colon along the length of the specimen with: ulceration, suppurative inflammation and ischaemic alteration of muscularis propria. Transmurally and within the coagulative necrosis were haematophagous amoebic trophozoites.

\section{Discussion}

Fulminant amoebic colitis is a rare complication of amoebiasis [2]. The disease is characterized by transmural inflammation, extensive colonic necrosis and perforation. The mortality is high, ranging from $60-100 \%$ in published series [2].

Although presented here are two cases of fulminant colitis where the aetiology was proven to be amoebiasis, it must be remembered that the differential diagnosis for fulminant colitis is broad [3]. Being a developing nation and in a sub-tropical environment, other infectious aetiologies would need to be considered. These would include viral aetiologies, such as Cytomegalovirus (CMV) and Herpes Simplex virus; and bacterial, such as Shigella, Salmonella and Campylobacter. Although ulcerative colitis is rare in the African population [4], when it does occur it has been reported to present with more extensive disease [5] and might have been a consideration in both these cases. Clostridium difficile can certainly present in a similar way, however this would usually occur in the setting of prolonged broad spectrum antibiotic use. Drug induced colitis and irradiation colitis can occur and would be suspected if there were relevant findings on history. Vasculitis-induced colitis would be a minor consideration in the patients presented here.

Since the patients in this series presented severely unwell with signs of generalized peritonitis, an initial operative approach was undertaken. If the presentation was less dramatic it may have been possible to initiate a period of intense medical management and taking a surgical approach if the response was poor. The diagnostic approach to a patient without peritonitis or objective evidence of perforation would include, laboratory investigations, imaging and possibly limited colonoscopy with biopsy.

It has been well described, that unlike other forms of fulminant colitis, fulminant amoebic colitis usually presents as an intraabdominal catastrophe mandating emergency laparotomy [6]. However, due to its rarity, the diagnosis of amoebic colitis is unlikely to be suspected pre-operatively. With the findings of friable "wet blotting paper" colon, multiple perforations and omental wraps, fulminant amoebic colitis must certainly be considered. This is especially true in the context of a patient living in or travelling from an endemic area. When disease is this extensive, excision of diseased colon and an exteriorising procedure offers patients the only chance of survival. This has been demonstrated in other case reports found in the literature, and represents current expert opinion [7-9]. Primary repair and anastomosis of bowel containing amoebae is likely to breakdown. In the second case presented here, excision margins were affected by the disease, it is presumed that here drug therapy heals any residual colitis.

At laparotomy, if the findings are that of an uncontaminated abdomen with adhesive wraps over diseased and possibly perforated colon, the preferred treatment is not resection as demonstrated by Luvuno [6]. Adhesive wraps are formed by omentum and peritoneal adhesions attracted to ischaemic foci of colon. Resectional surgery will dismantle these wraps and cause gross faecal spillage. In this situation performing an ileotomy and anterograde washout, followed by loop ileostomy to divert faecal stream has been demonstrated to have improved outcomes.

For reasons that are not clear, the patients in this series and others [7-9] failed to contain perforations and already had established faecal peritonitis. In this setting with extensive colonic necrosis, multiple perforations and gross contamination it is recommended to perform total/subtotal colectomy and exteriorization of proximal bowel (end ileostomy) with or without exteriorization of distal bowel (mucous fistula). This is followed by restoration of gastrointestinal continuity within 6 months.

\section{References}

1. Haque R (2007) Human intestinal parasites. J Health Popul Nutr 25: 387-391. [Crossref]

2. Aristizábal H, Acevedo J, Botero M (1991) Fulminant amebic colitis. World J Surg 15: 216-221. [Crossref]

3. Portela F, Lago P (2013) Fulminant colitis. Best Pract Res Clin Gastroenterol 27: $771-$ 782. [Crossref]

4. Segal I, Tim LO, Hamilton DG, Walker AR (1980) The rarity of ulcerative colitis in South African blacks. Am J Gastroenterol 74: 332-336. [Crossref]

5. Segal I (1988) Ulcerative colitis in a developing country of Africa: the Baragwanath experience of the first 46 patients. International Journal of Colorectal Disease 3: 222 225. [Crossref]

6. Luvuno FM (1990) Role of intraoperative prograde colonic lavage and a decompressive loop ileostomy in the management of transmural amoebic colitis. Br J Surg 77: 156159. [Crossref]

7. Ozdogan M, Baykal A, Aran O (2004) Amebic perforation of the colon: rare and frequently fatal complication. World J Surg 28: 926-929. [Crossref]

8. Ishida H IS, Murata N, Hashimoto D, Satoh K, Ohta S (2013) Fulminant amoebic colitis with perforation successfully treated by staged surgery: a case report. Journal of Gastroenterology 38: 92-96. [Crossref]

9. Gupta SS, Singh O, Shukla S, Raj MK (2009) Acute fulminant necrotizing amoebic colitis: a rare and fatal complication of amoebiasis: a case report. Cases Journal 2 : 6557. [Crossref]

Copyright: (C)2016 Wagener M. This is an open-access article distributed under the terms of the Creative Commons Attribution License, which permits unrestricted use, distribution, and reproduction in any medium, provided the original author and source are credited. 